\title{
BMJ Open Quality Using near-miss events to improve MRI safety in a large academic centre
}

\author{
Nirvani Goolsarran, ${ }^{1}$ Jose Martinez, ${ }^{2}$ Christine Garcia ${ }^{3}$
}

To cite: Goolsarran N Martinez J, Garcia C. Using near-miss events to improve MRI safety in a large academic centre. BMJ Open Quality 2019;8:e000593. doi:10.1136/ bmjoq-2018-000593

Received 30 November 2018 Revised 8 March 2019 Accepted 23 March 2019

\section{Check for updates}

(C) Author(s) (or their employer(s)) 2019. Re-use permitted under CC BY-NC. No commercial re-use. See rights and permissions. Published by BMJ.

${ }^{1}$ Department of Internal Medicine, Stony Brook University Hospital, Stony Brook, New York, USA

${ }^{2}$ NYU Langone Health, New York, New York, USA

${ }^{3}$ Department of Medicine, University of Pittsburgh Department of Medicine, Pittsburgh, Pennsylvania, USA

Correspondence to Dr Nirvani Goolsarran; nirvani.goolsarran@ stonybrookmedicine.edu

\begin{abstract}
Near-miss events represent an opportunity to identify and correct errors that jeopardise patient safety. The MRI environment poses potential safety threats and is frequently associated with near misses or adverse events related to improper safety screening for presence of cardiac pacemakers and other potential contraindications. At our institution, MRI safety screening lacked a formalised structure and standardisation; the process relied on a single-step safety screening process. As a result, we observed a significant number of near misses associated with improper MRI screening that resulted in 'close calls' in patients with incompatible metals implants. The purpose of this project was to use a quality improvement approach to analyse the near-miss pattern and create a multistep intervention to decrease the number of near misses associated with MRI screening and to ultimately decrease the potential for patient harm. Using the Plan-Do-Study-Act model, we decreased the number of MRI near misses from 22 to zero near misses in 1 year after implementation. The project demonstrates successful transformation of near misses to a never event: a reportable event that should never happen. The project also demonstrates the importance in targeting and prioritising a pattern of near misses, which are unplanned events that do not result in injury but had great potential to do so.
\end{abstract}

\section{PROBLEM (SMART AIM)}

MRI is a widely used diagnostic modality with 30 million scans being performed in the USA annually, according to the US Food and Drug Administration (FDA). ${ }^{1}$ Through electromagnetic field interactions, the MRI environment can potentially lead to dangerous consequences for patients. Improper safety screening for metal devices leads to potential hazards that include the following: dislodging medical or other metal implants, tissue heating, induced electrical currents, equipment or materials becoming dangerous missiles or projectiles, and potentially interrupting patient monitoring equipment. ${ }^{2}$ The development of a comprehensive and efficient screening procedure for potential contraindications is a critical component for patient safety.

Stony Brook University Hospital is an academic medical centre and serves as the tertiary care centre of Suffolk County, New York. At our institution, approximately 14
000 patients enter our in-house MRI scanners annually. Physician and mid-level providers typically order MRIs via our electronic medical record (EMR) system. A checkbox mechanism is a required field that is embedded in the EMR order to designate a patient having 'no contraindication to MRI'. The MRI imaging technicians then receive the electronic orders, and send a request for transportation of the patient to the MRI suite. Prior to placing the patient in the MRI scanning machine, a paper format safety screening questionnaire is conducted by the technician to ensure that there are no metal or potential contraindications to prohibit MRI scanning.

At our institution, there have been a total of 56 near-miss reports over the period of 1 year. A total of 22 out of $56(39 \%)$ incident reports were specifically related to unsafe MRI screening. Despite the use of a technician-administered screening questionnaire, patients with serious potential contraindications were physically transported to the MRI suite, and experienced 'close calls' near scans. Fortunately, due to the technician completion of the questionnaire, these 'near-miss' events were caught in time and prevented serious patient harm. Based on our incident reports, we recognised a pattern of near-miss events related to MRI screening that resulted in 'close calls' or 'good catches' by our technicians. Although no patient injury occurred, the aforementioned screening process presents an opportunity to investigate and analyse weaknesses in the system that resulted in the serious potential for harm. The aim of this project is to examine the near-miss pattern related to unsafe MRI screening, and develop an intervention to transform MRI near misses to never events in a 1-year period.

\section{BACKGROUND}

The WHO defines a near miss as 'an error that has the potential to cause an adverse event (patient harm) but fails to do so because of chance or because it is intercepted'. ${ }^{2}$ Near misses tend to occur frequently in during faulty processes or systems of care such as 
MRI ordering and screening. MRI is a widely used diagnostic modality with 30 million scans being performed in the USA annually, according to the US FDA. According to the FDA, while MRI is considered a safe modality, 'there is an underlying potential for injury to patients due to the strong electromagnetic (EM) fields used in MR scanning.' ${ }^{23}$

There is a paucity of the literature describing any standard approach to safely screen and order MRI imaging. To our knowledge, there is no standardised consensus on how to prevent unsafe MRI screening. Many institutions have developed questionnaire tools that need to be integrated into the workflow that is specific to the type of institution. The process is variable at every institution, and is often left at the discretion of the providers and leaders of the institution.

Unsafe MRI screening in patients with metal implantations can lead to patient morbidity and mortality, as a result of interactions of the MRI surrounding and the electric devices. The Joint Commission recommends a comprehensive MRI safety programme, which is essential in the overall safety programme in hospitals and medical care facilities. ${ }^{4}$

The Joint Commission and National Patient Safety Forum recommend that products or device events are viewed as never events that are preventable and reportable $^{5}$; hence, our goal was to transform MRI near misses to never events, a serious adverse event that should never happen or present itself for potential harm. This was achieved through the use of (1) a provider-driven safety screening checklist in EMR, (2) implementation of a computer hard stop in the EMR to prevent any bypass of the safety checklist or detected contraindications and (3) technician education on compliance of the MRI screening questionnaire. We aimed to transform MRI near misses to MRI never event, an event that should never happen.

\section{METHODS}

\section{Baseline Measurement}

As part of the pre-Plan-Do-Study-Act (PDSA) cycle, we reviewed incident reports from MRI safety threats for baseline measurement. We performed chart reviews to further contextualise the reported near misses. Data from the patient incident reporting system from January 2015 to 2016 showed a total of 22 near misses related to MRI screening. Analyses of the identified 22 near-misses revealed 11 out of $22(50 \%)$ that were patients with non-compatible MRI pacemakers. Further analysis of the 22 near misses showed that only 10 of 22 patients $(45 \%$ compliance rate) had completion of the technician MRI safety questionnaire form. Additionally, 22/22 (100\%) of the patients had completion of the checkbox for 'no contraindication for MRI' on the provider orders.

A multidisciplinary quality improvement (QI) team was formed to develop an understanding of the current MRI process of screening in order to identify key factors leading to improper screening. The team consisted of key stakeholders in the MRI ordering process including patient safety officers, resident trainees and attending physicians from radiology, neurology and medicine services, nurses and MRI technicians. An in-depth root cause analysis (RCA) was conducted to identify gaps/ areas of concern in the MRI screening process. The RCA was conducted over the course of three, 1-hour meetings, with stakeholders from radiology, neurology, emergency medicine, internal medicine and leadership. The Designated Institutional Officer and several Patient Safety/ Quality nurses led the RCA meetings. At the conclusion of the meeting, the identified gaps/areas of concern in the MRI screening process were outlined as follows:

1. Providers entered MRI orders into the EMR without completing verification of contraindications.

2. Prior to releasing the patient for transportation to the MRI suite, there was no nurse verification built into the workflow to confirm absence of MRI contraindications.

3. MRI technicians administered the questionnaire immediately before the patient has exam, often within a few feet of the MRI machine.

4. Although the paper screening safety questionnaire was readily available, compliance rates were low. Technicians were not consistently administering the screening form.

This multidisciplinary RCA showed that MRI screening process had several safety hazards, and identified points of possible vulnerability that could result in a potential system failure (presented in the Fishbone diagram in figure 1).

\section{DESIGN}

The data from the baseline measurement, in addition to discussion with stakeholders, revealed a number of significant areas with potential for improvement. The intervention consisted of multiple layers of safety checkpoints within the workflow.

First, we developed an EMR-based safety screening form that prompts providers to complete a safety screening checklist on initiation of an MRI order. Second, an EMR hard stop will prevent or cancel MRI orders if the checklist is incomplete or detect MRI incompatibility. The assigned nurse for the patient will verify completion of the form prior to patient transport. Finally, the technician safety screening questionnaire would serve as the final safety checkpoint for ensuring MRI compatibility and evaluation for MRI contraindications.

We employed the PDSA method of QI for this study. The study was conducted at Stony Brook University Hospital from September 2016 to September 2018.

\section{STRATEGY AND IMPROVEMENT CYCLES PDSA cycle 1}

Aim for PDSA cycle 1: To create and implement safety checkpoint $A$ in the EMR system to enforce screening form for potential MRI contraindications 


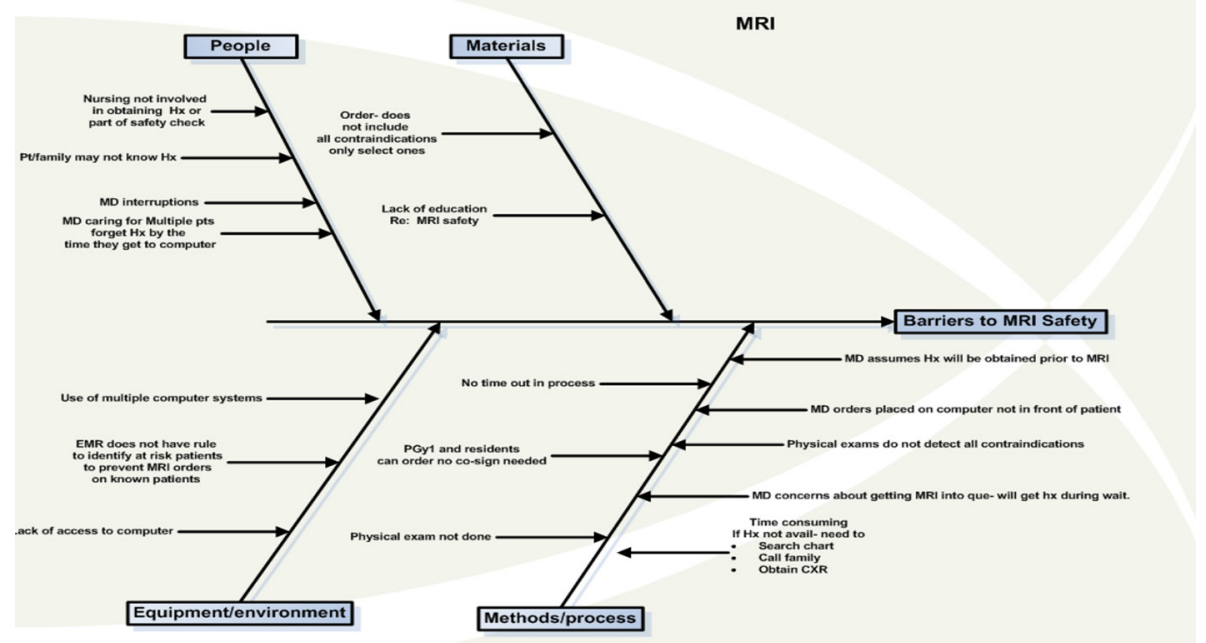

Figure 1 Root cause analysis of the MRI screening process prior to intervention. CXR, chest X-ray; EMR, electronic medical record; PGY, postgraduate year; MD, medical doctor; Hx, history.

The first PDSA cycle focused on the lack of provider accountability for MRI screening for potential contraindications with the patient. The QI team met with medical informatics team to design a safety screen prompt with a well-defined checklist of contraindications for all MRI orders. We created an EMR safety checklist in alignment with the Joint Commission recommendations for safe MRI screening. ${ }^{5}$ We then educated the residents, attendings and mid-level providers on how to use the new checklist format using electronic learning management modules we created. We specifically conducted in person education to the nurses to ensure completion of the safety checklist prior to releasing the patient for transport to MRI.

\section{PDSA cycle 2}

Aim for PDSA cycle 2: To create and implement safety checkpoint $B$ in the EMR system as a safety net to prevent bypass of the checklist or MRI orders with potential contraindications

Despite completion of the EMR safety screening form, we noticed that potential contraindications could still be missed if the providers or technicians are not specifically opening and viewing the form. Therefore, the aim of the second cycle was create to a safety net, an add-on to detect and prevent MRI orders with potential contraindications or incomplete safety forms. The EMR hard stop was built in to prompt the clinician to check for potential contraindications. As a forcing function, the MRI order is automatically cancelled if the alert is bypassed.

\section{PDSA cycle 3}

Aim for PDSA cycle 3: Revise the existing MRI technician questionnaire and create a final safety checkpoint $C$ to educate the technicians

The aim of PDSA cycle 3 was to specifically address the final safety checkpoint-the sharp end of an error or direct contact point with the patient just prior to MRI exam. We met and educated the MRI staff including the technicians. We edited the MRI safety questionnaire to create an identical version to the provider one in the EMR. All technicians received a power point didactic on the content and importance of completion of the questionnaire. The Director of MRI imaging also mandated this as part of the technician workflow. Additionally, reporting of all MRI safety threats was strongly championed and encouraged to assess the success of the new screening process. The overall flow diagram of the MRI screening process with checkpoints $\mathrm{A}, \mathrm{B}$ and $\mathrm{C}$ were reviewed with all providers (figure 2).

\section{RESULTS}

One-year baseline data were collected from January 2015 to January 2016, PDSA cycles 1-3 were implemented on March, April and May of 2016. We monitored data over time to monitor the success of the multistep MRI safety screening process. Post-intervention, we conducted review of our incident reports for MRI-related safety treats. One year post-intervention, we decreased the number of near misses related to MRI hazards from 22 out of 56 total near-missed reported (39\%) to zero out of $51(0 \%)$. To date, 2-years post-intervention, there are no near misses related to MRI safety hazards. Since there was no identified near-misses post-intervention, we cannot assess a true comparison between pre-compliance/post-compliance of safety questionnaire related to near misses. Therefore, to assess compliance of the safety questionnaire (safety check point $\mathrm{C}$ ), we reviewed a total of 1000 random selection of charts with MRI orders over a 6-month period post-intervention, we monitored for completion of final checkpoint screening questionnaire by the technicians. Post-intervention, a total of 957/1000 (95\%) had completion of final checkpoint $\mathrm{C}$ (figure 3).

\section{Lessons and limitations}

There were several limitations to our project. First, there were a low number of incident reports conducted by staff 


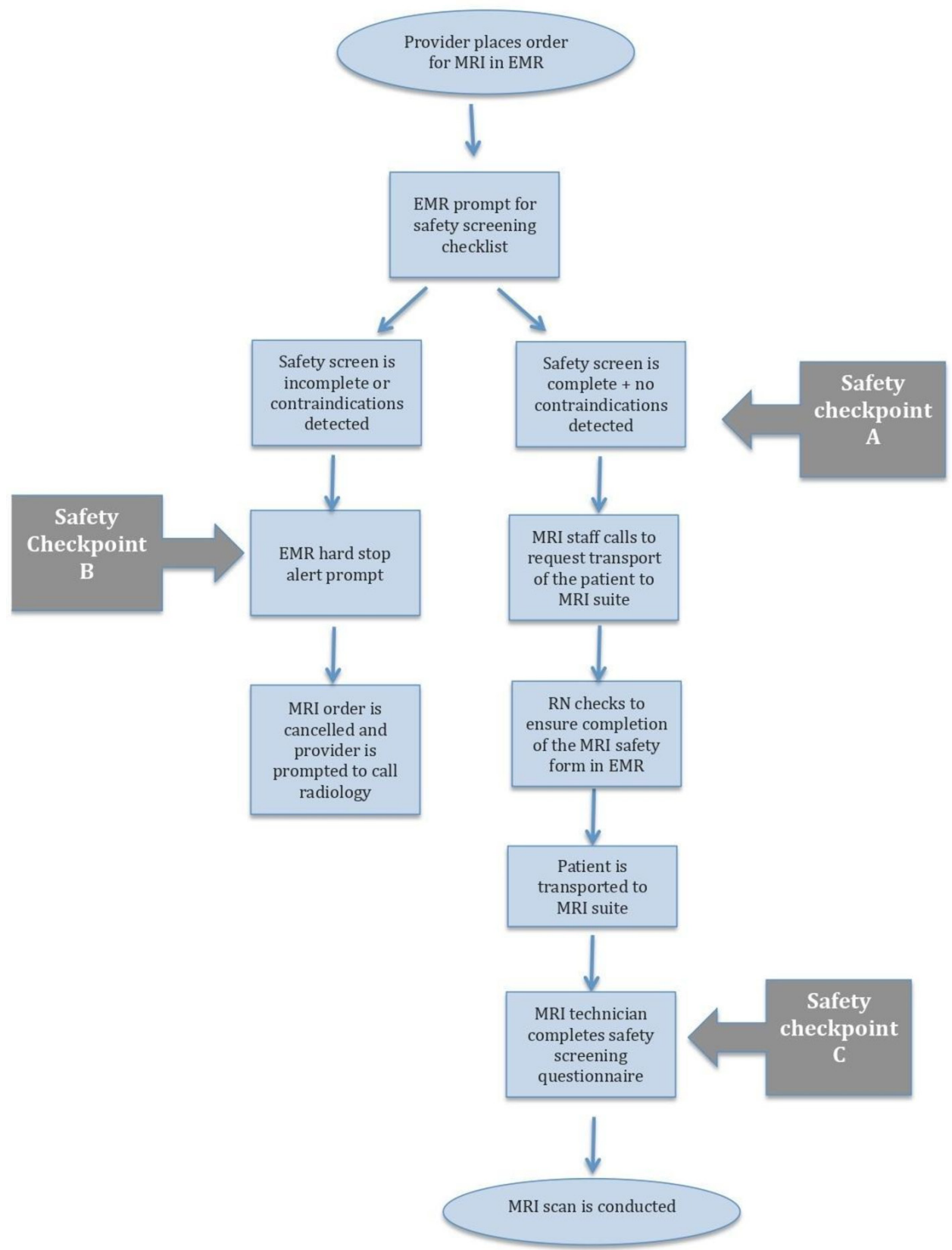

Figure 2 Flow diagram of MRI ordering process. EMR: electronic medical record; RN: registered nurse.

members at our institution, resulting in a small sample size and limited evaluation of the baseline data. This study relies on incident reports to detect MRI hazards during the pre-period and post-period of the study. Therefore, the number of near misses during pre-evaluation/post-evaluation may be underestimated given the lack of incident reports. At our institution, around 14 000 patients annually undergo MRI scans. The use of our incident reports was the only way for us to analyse true near misses without having to do direct observations for each MRI scan for a prolonged study period, or alternatively, complete a retrospective chart search which may not reveal near misses.

Second, due to time constraints, we implemented PDSA cycles 1-3 within 3 months. It is difficult to individually assess the effectiveness of each of QI cycle. Ideally, we should have assessed each cycle over a longer period of time to better assess the overall effects of the multistep intervention. Generally, forcing functions, such as the EMR hardstop for MRI orders, are more effective for error reduction ${ }^{6}$; however, perhaps the sustained success of this project is a result of our multicomponent structure with education, standardisation and forcing functions. Another limitation is the lack of assessment of learner's knowledge following education of the importance of MRI safety and the ordering process. Our intervention involved education of the residents, attendings, advanced care practitioners and MRI technicians; therefore, evaluation of change in learner's knowledge should have supplement behavioural changes related to MRI safety. 


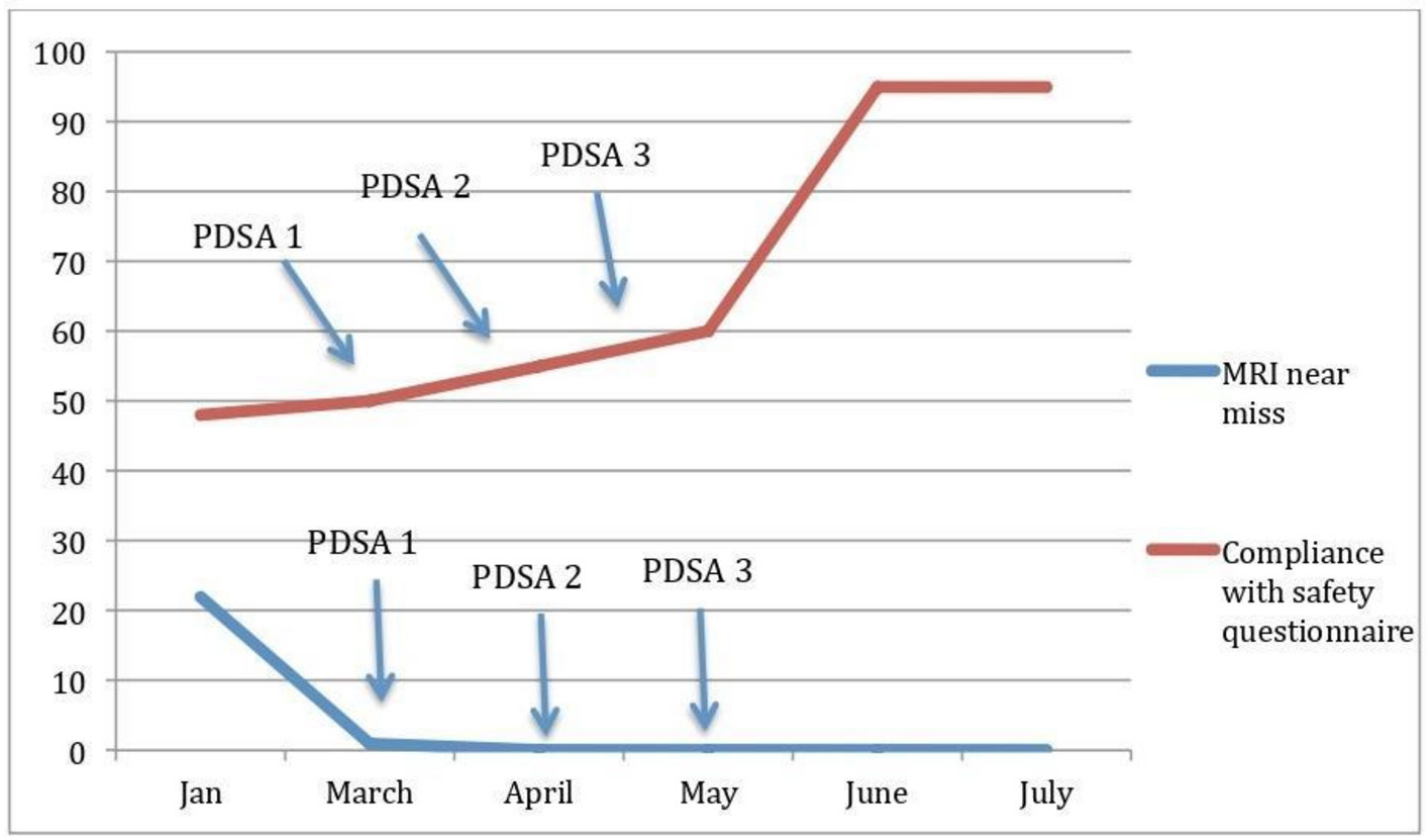

Figure 3 Results over time (PDSA cycles 1-3). PDSA, Plan-Do-Study-Act.

There were many lessons learnt from this intervention. Repeat didactics and website postings were necessary to educate and emphasise the importance of the screening questionnaire to the technicians. Specifically, we found that education of the purpose behind the intervention and potential consequences of MRI incompatibility burns were very useful strategies to target provider buy-in. It was critical to have leadership buy-in from the institution safety officers and Chief Medical Officer endorsement.

In developing an EMR intervention, we learnt that a checklist of contraindications is crucial and should be embedded in the actual EMR order. Lastly, through our workflow analysis, we learnt that the MRI screening process is not a single step or single provider process, it includes close collaboration between the providers, nursing staff and MRI technicians in order to create a successful intervention.

\section{CONCLUSIONS}

Our QI project demonstrates the need for multidisciplinary collaboration in developing a safe MRI screening process. Our project successfully transformed a nearmiss pattern related to MRI hazards to a never event, with sustainable results over 2 years. Ultimately, the MRI screening process is complex, and should ideally be a multistep process with several safety checkpoints to prevent lapses or weaknesses in the system. We believe that our process of developing a multistep MRI screening process by using QI methodology can be duplicated at other hospitals to reduce the risk of potential harm to our patients.
Acknowledgements The authors would like to thank Susan Robbins, the chief quality informatics-nursing officer for leading the RCA group.

Contributors NG was responsible for the overall content of the manuscript, she conceptualised the design and drafted the original manuscript. JM monitored and tracked the data, and designed the results of the study. CG revised and edited the final version of the manuscript.

Funding The authors have not declared a specific grant for this research from any funding agency in the public, commercial or not-for-profit sectors.

Competing interests None declared.

Patient consent for publication Not required.

Ethics approval The Institutional Review Board at Stony Brook University Hospital, NY deemed this study as exempt as an educational initiative. IRB acknowledgement \# 717726-1.

Provenance and peer review Not commissioned; externally peer reviewed. Open access This is an open access article distributed in accordance with the Creative Commons Attribution Non Commercial (CC BY-NC 4.0) license, which permits others to distribute, remix, adapt, build upon this work non-commercially, and license their derivative works on different terms, provided the original work is properly cited, appropriate credit is given, any changes made indicated, and the use is non-commercial. See: http://creativecommons.org/licenses/by-nc/4.0/.

\section{REFERENCES}

1. U.S. Department of health and Human services. Food and drug administration. Magnetic resonance imaging (MRI) safety, Aug. 1, 2016. Available: www.mrisafety.comURL:www.mrisafety.com

2. Guidelines for screening patients for MRI procedures and individuals for the MRI environment. Available: www.mrisafety.com

3. The Joint Commission, division of healthcare improvement: strong MRI programs prevent safety issues, Issue 31, March 2017. Available: https://www.jointcommission.org/assets/1/23/Quick_Safety_Issue_ 31_2017_MRI_safety.pdf

4. Available: http://www.qualityforum.org/Topics/SREs/List_of_SREs. aspx

5. Available: https://www.jointcommission.org/assets/1/23/Quick Safety_Issue_31_2017_MRI_safety.pdf

6. Gosbee JW, Gosbee LL, eds. Human Factors Engineering to Improve Patient Safety. Oakbrook IL: Joint Commission Resources, 2005. 